\section{A Switching Algorithm for Global Exponential Stabilization of Uncertain Chained Systems}

\author{
Zairong Xi, Gang Feng, Z. P. Jiang, and Daizhan Cheng
}

\begin{abstract}
This note deals with chained form systems with strongly nonlinear unmodeled dynamics and external disturbances. The objective is to design a robust nonlinear state feedback law such that the closed-loop system is globally $\mathcal{K}$-exponentially stable. We propose a novel switching control strategy involving the use of input/state scaling and integrator backstepping. The new features of our controllers include the ability to achieve Lyapunov stability, exponential convergence, and robustness to a set of uncertain drift terms.
\end{abstract}

Index Terms-Backstepping, chained form systems, exponential stabilization, input-state scaling, Lyapunov stability, robustness.

\section{INTRODUCTION}

Over the past decade, the control and stabilization of nonholonomic systems has formed an active area within the nonlinear control community; see, for example, the recent survey papers [5], [11], and the references cited therein for an interesting introduction to this quickly expanding area. This flow of research activity has been mainly triggered by the well-known 1983 paper by Brockett [4], where a necessary condition for asymptotic stabilization is stated. One of the consequences of the necessary condition is that a nonholonomic system is not stabilizable by stationary continuous state feedback. To overcome this impossibility, several interesting and fundamentally nonlinear approaches have been proposed. Examples of these approaches are open-loop periodic steering control, either smooth or continuous time-varying control, and discontinuous feedback control; see, for example, [1]-[3], [6], [7], [9], [10], [13], and [15]-[20].

It should be noted that the majority of these constructive methods have been developed around an important class of driftless nonholonomic systems in chained form, which was brought to the literature by [18]. As explained and illustrated in [11], [18], and the references therein, many nonlinear mechanical systems with nonholonomic constraints on velocities can be transformed, either locally or globally, to chained form systems via coordinates and state-feedback transformation. For instance, we have seen such examples as tricycle-type mobile robots, cars towing several trailers, the knife edge, a vertical rolling wheel, and a rigid spacecraft with two torque actuators.

Manuscript received January 21, 2003; revised May 20, 2003. Recommended by Associate Editor J. Huang. This work was supported in part by a Grant from the Research Grants Council of the Hong Kong Special Administrative Region, China under Project CityU 1024/02E, the National Natural Science Foundation of China and the Institute of Systems Science, Academy of Mathematics and Systems Science, and the Chinese Academy of Sciences. The work of Z. P. Jiang was supported in part by the National Science Foundation under Grant ECS0093176 and Grant INT-9987317.

$\mathrm{Z}$. Xi is with the Laboratory of Systems and Control, Institute of Systems Science, Academy of Mathematics and Systems Science, Chinese Academy of Sciences, Beijing 100080, China, and also with the Department of Manufacture Engineering and Engineering Management, The City University of Hong Kong, Kowloon Tong, Hong Kong (e-mail: mezrxi@ cityu.edu.hk).

G. Feng is with the Department of Manufacture Engineering and Engineering Management, The City University of Hong Kong, Kowloon Tong, Hong Kong (e-mail: megfeng@cityu.edu.hk).

Z. P. Jiang is with the Department of Electrical and Computer Engineering, Polytechnic University, Brooklyn, NY 11201 USA.

D. Cheng is with the Laboratory of Systems and Control, Institute of Systems Science, Academy of Mathematics and Systems Science, Chinese Academy of Sciences, Beijing 100080, China.

Digital Object Identifier 10.1109/TAC.2003.817937
As is well known in the literature on nonholonomic control systems, a smooth time-varying state-feedback law can be applied to achieve asymptotic stabilization but fails to meet the requirement of exponential convergence. However, exponential convergence is an important performance characteristic for practical applications. To date, several important steps have been made toward the design of a continuous time-varying and/or discontinuous feedback law guaranteeing the exponential regulation of nonholonomic systems in chained form [2], [10], [16], and [20]. Two types of control laws-discontinuous state feedback and time-varying feedback - have been frequently used in the recent literature to obtain an exponential rate of convergence for nonholonomic control systems (see, for instance, [1], [2], [6], [10], [14], [16], and [20]). However, the closed-loop systems are not Lyapunov stable. Marchand and Alamir [15] obtained Lyapunov stability and exponential rate of convergence in the absence of disturbances. Since their result depends on a Riccati equation, it could not be easily extended, if not impossible, to the occurrence of uncertain disturbances [15].

The purpose of this note is to obtain both robust global exponential regulation and Lyapunov stability for a class of disturbed nonlinear chained systems without imposing any restriction on the system order and the growth of the uncertain nonlinearities. The contribution of the note is twofold. We propose a systematic control design procedure to construct a switching robust nonlinear control law which not only solves the global exponential regulation problem, but also Lyapunov stability problem for all plants in the considered class, including the ideal chained system. For the Lyapunov stability with global exponential regulation, to the best of our knowledge, there is still no robustification tool for nonholonomic systems design.

The remainder of this note is organized as follows. In Section II, the class of nonholonomic systems with strongly nonlinear disturbances is introduced and the problem of global exponential stabilization is formulated. Section III first presents the input-state scaling technique and the backstepping design procedure and then a switching control strategy. In Section IV, we illustrate our novel control design methodology via a practical nonholonomic system with disturbances. The numerical simulations testify to the effectiveness and robustness aspects of the proposed robustification tool. Finally, some conclusions are given in Section V.

\section{PROBLEM Formulation}

The purpose of this note is to consider a perturbed version of the chained form [10]

$$
\left\{\begin{array}{l}
\dot{x}_{0}=d_{0}(t) u_{0}+x_{0} \phi_{0}^{d}\left(t, x_{0}\right) \\
\dot{x}_{1}=d_{1}(t) x_{2} u_{0}+\phi_{1}^{d}\left(t, x_{0}, x, u_{0}\right) \\
\vdots \\
\dot{x}_{n-2}=d_{n-2}(t) x_{n-1} u_{0}+\phi_{n-2}^{d}\left(t, x_{0}, x, u_{0}\right) \\
\dot{x}_{n-1}=d_{n-1}(t) u+\phi_{n-1}^{d}\left(t, x_{0}, x, u_{0}\right)
\end{array}\right.
$$

where $x=\left(x_{1}, \ldots, x_{n-1}\right) \in R^{n-1}, x_{0} \in R$ the functions $d_{i}$ ' $\mathrm{s}$ and $\phi_{i}^{d}$ 's denote the possible modeling error and neglected dynamics. Throughout this note, the following assumptions will be required.

Assumption 1: For every $0 \leq i \leq n-1$, there are (known) positive constants $c_{i 1}$ and $c_{i 2}$ such that

$$
c_{i 1} \leq d_{i}(t) \leq c_{i 2} \quad \forall t \geq 0 .
$$


Assumption 2: For every $0 \leq i \leq n-1$, there is a (known) smooth nonnegative functions $\phi_{i}$ that satisfies the inequalities

$$
\begin{aligned}
\left|\phi_{0}^{d}\left(t, x_{0}\right)\right| & \leq \phi_{0}\left(x_{0}\right) \\
\left|\phi_{i}^{d}\left(t, x_{0}, x, u_{0}\right)\right| & \leq\left|\left(x_{1}, \ldots x_{i}\right)\right| \phi_{i}\left(x_{1}, \ldots x_{i}, u_{0}\right)
\end{aligned}
$$

for all $\left(t, x_{0}, x, u_{0}\right) \in R_{+} \times R \times R^{n-1} \times R$.

As explained and illustrated in [10], the structural triangularity condition in Assumption 2 is a quite common assumption in the framework of robust/adaptive nonlinear control [12].

We first recall a notion of $\mathcal{K}$-exponential stability from [20], which reduces to exponential stability in the Lyapunov sense when the function $\gamma$ is a linear function.

Definition 1: A system of the form $\sum: \dot{x}=f(x)$ with $x \in R^{n}$ is said to be globally $\mathcal{K}$-exponentially stable (GES) if there exist a positive constant $\lambda$ and a function $\gamma$ of class $\mathcal{K}$ such that $\forall x(0) \in R^{n}, \forall t \geq 0$

$$
|x(t)| \leq \gamma(|x(0)|) e^{-\lambda t} \quad \forall t \geq 0 .
$$

This note aims to find explicit controllers

$$
u_{0}=\mu_{0}\left(x_{0}, x\right) \quad u=\mu\left(x_{0}, x\right)
$$

that globally $\mathcal{K}$-exponentially stabilize all systems (1) satisfying Assumptions 1 and 2. A main difference with [1], [2], and [10] is that we are interested in achieving stability properties in the sense of Lyapunov.

On the basis of Assumptions 1 and 2, we are led to choose the control law $u_{0}$ as

$$
u_{0}=-\lambda_{0} x_{0}-\frac{1}{c_{01}} x_{0} \phi_{0}\left(x_{0}\right)
$$

where $\lambda_{0}>0$ is a positive design parameter. As a result, the following lemma can be established by considering the Lyapunov function candidate $V_{0}=(1 / 2) x_{0}^{2}$ and by applying directly the Gronwall Lemma (cf. [10]).

Lemma 1: For any initial instant $t_{0} \geq 0$ and any initial condition $x_{0}\left(t_{0}\right) \in R$, the corresponding solution $x_{0}(t)$ exists for each $t \geq t_{0}$ and satisfies $\lim _{t \rightarrow \infty} x_{0}(t)=0$. Furthermore, if $x_{0}\left(t_{0}\right) \neq 0$ then $x_{0}(t) \neq 0$ for all $t \geq t_{0}$.

Notice that the forward invariance property proved in Lemma 1 will be used in the controller design and stability analysis in the next section.

\section{CONTROLler DESIGN}

\section{A. Input-State Scaling and Backstepping Design}

Introduce an input-state scaling discontinuous transformation defined by [6] and [15]

$$
\xi_{i}=\frac{x_{i}}{u_{0}^{n-(i+1)}}, \quad 1 \leq i \leq n-1
$$

Under the new $\xi$-coordinates, the $x$-system is transformed into

$$
\left\{\begin{array}{l}
\dot{\xi}_{1}=d_{1}(t) \xi_{2}-(n-2) \xi_{1} \frac{\dot{u}_{0}}{u_{0}}+\frac{\phi_{1}^{d}\left(t, x_{0}, x, u_{0}\right)}{u_{0}^{n-2}} \\
\dot{\xi}_{2}=d_{2}(t) \xi_{3}-(n-3) \xi_{2} \frac{\dot{u}_{0}}{u_{0}}+\frac{\phi_{2}^{d}\left(t, x_{0}, x, u_{0}\right)}{u_{0}^{n-3}} \\
\vdots \\
\dot{\xi}_{n-2}=d_{n-2}(t) \xi_{n-1}-\xi_{n-2} \frac{\dot{u}_{0}}{u_{0}}+\frac{\phi_{n-2}^{d}\left(t, x_{0}, x, u_{0}\right)}{u_{0}} \\
\dot{\xi}_{n-1}=d_{n-1}(t) u+\phi_{n-1}^{d}\left(t, x_{0}, x, u_{0}\right)
\end{array} .\right.
$$

The inherently triangular structure of (1) suggests that we should design the control inputs $u_{0}$ and $u$ in two separate stages.

Assumption 3: Assume that $u_{0}: R \rightarrow R$ is a continuous, almost everywhere differentiable function, with the following properties:

P1) for all $t \geq 0, u_{0}(t) \neq 0$;

P2) for almost all $t \geq 0,\left|d u_{0} / d t\right| \leq \bar{\phi}_{0}\left(x_{0}\right)\left|u_{0}(t)\right|$, where $\bar{\phi}_{0}\left(x_{0}\right)$ is a known nonnegative function.

If $u_{0}$ vanishes, $x$ clearly becomes uncontrollable. P1) avoids this loss of controllability. P2) is only convenient for the control design. From Section II, it is known that $u_{0}=-\left(\lambda_{0}+\left(\phi_{0} / c_{01}\right)\right) x_{0}$ fulfills Assumption 3 provided $x_{0}\left(t_{0}\right) \neq 0$.

In the remainder of this section, we focus on designing the control input $u$ provided that Assumptions 1-3 are satisfied.

According to Assumption 3, the discontinuous state transformation (6) is applicable because $u_{0}(t) \neq 0$ for every $t \geq t_{0}$. The design of the control input $u$ will be based on an application of the common backstepping method to the transformed system (7). Indeed, (7) can be written in the more compact form

$$
\left\{\begin{array}{l}
\dot{\xi}_{1}=d_{1}(t) \xi_{2}+\Phi_{1}^{d}\left(t, x_{0}, x, u_{0}\right) \\
\dot{\xi}_{2}=d_{2}(t) \xi_{3}+\Phi_{2}^{d}\left(t, x_{0}, x, u_{0}\right) \\
\vdots \\
\dot{\xi}_{n-2}=d_{n-2}(t) \xi_{n-1}+\Phi_{n-2}^{d}\left(t, x_{0}, x, u_{0}\right) \\
\dot{\xi}_{n-1}=d_{n-1}(t) u+\Phi_{n-1}^{d}\left(t, x_{0}, x, u_{0}\right)
\end{array}\right.
$$

where, for each $1 \leq i \leq n-1$

$$
\Phi_{i}^{d}=\frac{\phi_{i}^{d}\left(t, x_{0}, x, u_{0}\right)}{u_{0}^{n-(i+1)}}-(n-(i+1)) \xi_{i} \frac{\dot{u}_{0}}{u_{0}} .
$$

Lemma 2: For each $1 \leq i \leq n-1$, there exists a smooth nonnegative function $\bar{\Phi}_{i}$ such that

$\left|\Phi_{i}^{d}\left(t, x_{0}, x, u_{0}\right)\right| \leq\left|\left(\xi_{1}, \cdots, \xi_{i}\right)\right| \bar{\Phi}_{i}\left(x_{0}, \xi_{1}, \cdots, \xi_{i}, u_{0}\right)$.

Proof: In view of (6), Assumptions 1-3, we have

$$
\begin{aligned}
\left|\Phi_{i}^{d}\left(t, x_{0}, x, u_{0}\right)\right| \leq & \frac{\left|\left(x_{1}, \ldots, x_{i}\right)\right|}{\left|u_{0}^{n-(i+1)}\right|} \phi_{i}\left(x_{1}, \cdots, x_{i}, u_{0}\right) \\
& +(n-(i+1)) \bar{\phi}_{0}\left(x_{0}\right)\left|\xi_{i}\right| \\
\leq & \left|\left(\left(u_{0}\right)^{i-1} \xi_{1}, \cdots, \xi_{i}\right)\right| \\
& \times \phi_{i}\left(\left(u_{0}\right)^{n-2} \xi_{1}, \ldots,\left(u_{0}\right)^{n-(i+1)} \xi_{i}, u_{0}\right) \\
& +(n-(i+1)) \bar{\phi}_{0}\left(x_{0}\right)\left|\xi_{i}\right| .
\end{aligned}
$$

Therefore, the proof of Lemma 2 is completed.

Thanks to Lemma 2, (8) satisfies the "lower-triangularity" condition and therefore, the systematic controller design for $u$ can be obtained using so-called backstepping methods [6], [10], [12].

Step 1: Let us begin with the scalar $\xi_{1}$ subsystem of (8)

$$
\dot{\xi}_{1}=d_{1}(t) \xi_{2}+\Phi_{1}^{d}\left(t, x_{0}, x, u_{0}\right)
$$

where $\xi_{2}$ is regarded as the virtual control input. Let $z_{1}=\xi_{1}$ and introduce the Lyapunov function $V_{1}=(1 / 2) z_{1}^{2}$. Using Lemma 2, the time derivative of $V_{1}$ along the solutions of (8) satisfies

$$
\dot{V}_{1} \leq d_{1}(t) z_{1} \xi_{2}+z_{1}^{2} \bar{\Phi}_{1}\left(x_{0}, z_{1}, u_{0}\right)
$$


Then, with Assumption 1, we are led to introduce a virtual control function $\alpha_{1}$ and a new variable $z_{2}$

$$
\begin{aligned}
\alpha_{1}\left(x_{0}, z_{1}, u_{0}\right) & =-\lambda_{1} z_{1}-\frac{1}{c_{11}} \varphi_{1}\left(x_{0}, z_{1}, u_{0}\right) z_{1} \\
z_{2} & =\xi_{2}-\alpha_{1}\left(x_{0}, z_{1}, u_{0}\right)
\end{aligned}
$$

where $\lambda_{1}$ is a positive design parameter and $\varphi_{1}=\bar{\Phi}_{1}$ is a smooth nonnegative function. Consequently, (11) implies

$$
\dot{V}_{1} \leq-\lambda_{1} d_{1}(t) z_{1}^{2}+d_{1}(t) z_{1} z_{2}
$$

Note that $\alpha_{1}$ is a smooth function satisfying

$$
\alpha_{1}\left(x_{0}, 0, u_{0}\right)=0 \quad \forall x_{0} \in R
$$

Step $i(2 \leq i \leq n-2)$ : As in [10] and Step 1, consider the Lyapunov function candidate $V_{i}=V_{i-1}\left(z_{1}, \ldots, z_{i-1}\right)+(1 / 2) z_{i}^{2}$. Therefore, we can choose a virtual control function $\alpha_{i}$ and a new variable $z_{i+1}$ as follows:

$$
\begin{aligned}
\alpha_{i} & =-\lambda_{i} z_{i}-\sum_{j=1}^{i} \frac{1}{c_{j 1}} \varphi_{i j}\left(x_{0}, z_{1}, \cdots, z_{i}, u_{0}\right) z_{j} \\
z_{i+1} & =\xi_{i+1}-\alpha_{i}
\end{aligned}
$$

where $\varphi_{i j}\left(x_{0}, z_{1}, \cdots, z_{i}, u_{0}\right)$ are some nonnegative function derived from backstepping, such that

$$
\dot{V}_{i} \leq-\sum_{j=1}^{i}\left(\lambda_{j} d_{j}(t)-i+j\right) z_{j}^{2}+d_{i}(t) z_{i} z_{i+1} .
$$

Step $n-1$ : At this last step, consider the whole $\xi$-system (8) where the true input $u$ is to be designed on the basis of the virtual control functions $\alpha_{i}$ 's. To this end, consider a positive-definite and radially unbounded Lyapunov function

$$
V_{n-1}=V_{n-2}\left(z_{1}, \ldots, z_{n-2}\right)+\frac{1}{2} z_{n-1}^{2} .
$$

As in [6], [10], and [12], it is easy to know that some smooth nonnegative function $\varphi_{(n-1) j}\left(x_{0}, z_{1}, \ldots, z_{n-1}, u_{0}\right)(j=1, \cdots, n-$ 1) can be found such that along the solutions of (8)

$$
\dot{V}_{n-1} \leq-\sum_{j=1}^{n-1}\left(\lambda_{j} d_{j}(t)-n+1+j\right) z_{j}^{2}
$$

when choosing the control law $u$ as

$$
\begin{aligned}
u= & \alpha_{n-1}\left(x_{0}, z_{1}, \ldots, z_{n-1}, u_{0}\right) \\
= & -\lambda_{n-1} z_{n-1} \\
& -\sum_{j=1}^{n-1} \frac{1}{c_{j 1}} z_{j} \varphi_{(n-1) j}\left(x_{0}, z_{1}, \ldots, z_{n-1}, u_{0}\right) .
\end{aligned}
$$

Therefore, the following theorem can be obtained.

Theorem 1: Under Assumptions 1-3, if parameters $\lambda_{i}$ 's satisfies

$$
\lambda=\min \left\{\lambda_{j} c_{j 1}-n+1+j \mid, \quad j=1, \ldots, n-1\right\}>0
$$

then the aforementioned control strategy (13) yields that the $x$-subsystem of uncertain system (1) with $x=\left(x_{1}, \cdots, x_{n-1}\right)$ is well defined and is globally $\mathcal{K}$-exponentially stabilized at the origin.

Proof: Let $z=\left(z_{1}, \ldots, z_{n-1}\right)$. According to (12), we have

$$
\dot{V}_{n-1} \leq-\lambda V_{n-1}
$$

which implies

$$
|z(t)| \leq|z(0)| e^{-\lambda t}, \quad t \geq 0
$$

Then [10]

$$
|\xi(t)| \leq \gamma\left(\left|\left(x_{0}(0), \xi(0), u_{0}(0)\right)\right|\right) e^{-\varepsilon t}, \quad t \geq 0
$$

where $\varepsilon>0, \xi=\left(\xi_{1}, \ldots, \xi_{n-1}\right)$ and $\gamma$ is a class- $\mathcal{K}$ function.

Hence, (3) follows readily from (6).

As a particular case of Theorem 1, one has the following.

Theorem 2: Assumptions 1 and 2, if $x_{0}(0) \neq 0$ and parameters $\lambda_{i}$ 's satisfy

$$
\lambda=\min \left\{\lambda_{j} c_{j 1}-n+1+j \mid, \quad j=1, \ldots, n-1\right\}>0
$$

then the aforementioned control strategy (5) and (13) yields that the uncertain system (1) is globally exponentially regulated at the origin in the sense that all the trajectories satisfy (3).

Proof: We only need to verify that

$$
\left|\frac{\dot{u}_{0}}{u_{0}}\right| \leq \bar{\phi}_{0}\left(x_{0}\right)
$$

In fact, $u_{0}=-\lambda_{0} x_{0}-\left(1 / c_{01}\right) x_{0} \phi_{0}\left(x_{0}\right)$, then

$$
\dot{u}_{0}=-\left(\lambda_{0}+\frac{1}{c_{01}} \phi_{0}\left(x_{0}\right)+\frac{1}{c_{01}} x_{0} \phi_{0}^{\prime}\left(x_{0}\right)\right)\left(d_{0} u_{0}+x_{0} \phi_{0}^{d}\right) .
$$

So

$$
\begin{aligned}
\left|\frac{\dot{u}_{0}}{u_{0}}\right| \leq\left(c_{02}+\right. & \left.\frac{\phi_{0}\left(x_{0}\right)}{\lambda_{0}}\right) \\
& \times\left(\lambda_{0}+\frac{1}{c_{01}} \phi_{0}\left(x_{0}\right)+\frac{1}{2 c_{01}}\left(x_{0}^{2}+\left(\phi_{0}^{\prime}\left(x_{0}\right)\right)^{2}\right)\right) .
\end{aligned}
$$

\section{B. Switching Scheme}

In the preceding discussions, we have given the controller expressions (5) and (13) for $u_{0}$ and $u$ of (1) if the starting point of the $x_{0}$ state component is not zero, i.e., $x_{0}\left(t_{0}\right) \neq 0$. Without loss of generality, we can assume that $t_{0}=0$. Now, we discuss how to select the control laws $u_{0}$ and $u$ when $x_{0}(0)=0$.

The purpose of this section is to answer this question by proposing a globally exponentially stabilizing static state feedback. Roughly speaking, when the initial state is $x_{0}(0)=0$ and $x(0) \neq 0$ we first use an "almost" (nonzero) constant action $u_{0}$ and the corresponding control $u$ that is designed based on a discontinuous coordinates transformation of the form (6) and backstepping technique to drive the state $x_{0}$ away from 0 in a short time duration $\left[0, t_{s}\right)$, which depends only on initial point. Then, the almost constant feedback law is switched to an exponential regulator which is also based on a discontinuous coordinates transformation of the form (6) and backstepping technique. 
However, in the present situation, the presence of nonlinear uncertain functions $\phi_{i}^{d}(0 \leq i \leq n-1)$ may lead some solutions to blow up before the given switching time $t_{s}$. To prevent this phenomenon from happening, the following switching control strategy for both control inputs $u_{0}$ and $u$ is proposed.

Theorem 3: Let

- $\beta, \delta, \lambda_{0}, \epsilon$ and $\lambda_{i}(1 \leq i \leq n-1)$ be strictly positive real constants so that

$\lambda=\min \left\{\lambda_{j} c_{j 1}-n+1+j \mid, \quad j=1, \ldots, n-1\right\}>0 ;$

- $T_{0}$ be defined in $[0, \infty)$ by

$$
T_{0}(s)=\max _{\tau \in[-s, s]}\left\{\epsilon+\phi_{0}(\tau)\right\}
$$

which is a nondecreasing continuous function;

- $\Gamma=\{(0, x):|x| \neq 0\}$;

Then, the following static discontinuous feedback law globally $\mathcal{K}$-exponentially stabilizes the uncertain chained form system (1). Moreover, the feedback law is bounded.

i) When $\left(x_{0}(0), x(0)\right)=(0, x(0)) \in \Gamma$,

$$
\begin{aligned}
& u_{0}(t)= \begin{cases}\beta-\frac{\phi_{0}}{c_{01}} x_{0}, & \text { if } t<t_{s}(|x(0)|) \\
-\left(\lambda_{0}+\frac{\phi_{0}}{c_{01}}\right) x_{0}, & \text { if } t \geq t_{s}(|x(0)|)\end{cases} \\
& u(t)=\alpha_{n-1}\left(x_{0}, x, u_{0}\right) \quad \forall t>0
\end{aligned}
$$

where $t_{s}(|x(0)|)=\min \left\{\delta,\left(c_{01} /\left(2 c_{02} T_{0}\left(c_{02} \beta|x(0)|\right)\right)\right)\right.$, $|x(0)|\}$.

ii) When $\left(x_{0}(0), x(0)\right)=(0,0)$

$$
\begin{aligned}
u_{0} & =0 \\
u & =0 .
\end{aligned}
$$

iii) When $\left(x_{0}(0), x(0)\right) \notin \Gamma \cup\{(0,0)\}$

$$
\begin{gathered}
u_{0}=-\left(\lambda_{0}+\frac{\phi_{0}}{c_{01}}\right) x_{0} \\
u=\alpha_{n-1}\left(x_{0}, x, u_{0}\right) .
\end{gathered}
$$

In order to prove Theorem 3, the following Lemma is needed.

Lemma 3: Consider the uncertain differential equation

$$
\dot{x}_{0}=d_{0}(t) u_{0}+x_{0} \phi_{0}^{d}\left(t, x_{0}\right), \quad x_{0} \in R, x_{0}(0)=0 .
$$

If $\left|\phi_{0}^{d}\left(t, x_{0}\right)\right| \leq \phi_{0}\left(x_{0}\right)$ and $0<c_{01} \leq d_{0}(t) \leq c_{02}$, then the closed-loop system with $u_{0}=\beta-x_{0}\left(\left(\phi_{0}\left(x_{0}\right)\right) / c_{01}\right)$ has the following properties:

$$
\left|x_{0}(t)\right| \leq c_{02} \beta t, \quad t>0
$$

and

$$
x_{0}(t)>0, \quad t>0
$$

where $\beta>0$.

Proof: It is easy to see that $u_{0}(t)$ is continuous and $u_{0}(0)=\beta>$ 0 . So $\dot{x}_{0}(0)>0$ and $x_{0}(t)$ is strictly increasing in a small time duration $\left[0, t_{1}\right]$. Then, it is not difficult to know that $x_{0}(t) \geq 0$ for all $t>0$.
Differentiating $V_{0}=x_{0}^{2}$ along (20), we obtain

$$
\begin{aligned}
\frac{d V_{0}}{d t} & =2 x_{0}\left(d_{0}(t) u_{0}+x_{0} \phi_{0}^{d}\left(t, x_{0}\right)\right) \\
& \leq 2 c_{02} \beta x_{0} \\
& =2 c_{02} \beta \sqrt{V_{0}}
\end{aligned}
$$

Then

$$
\sqrt{V_{0}(t)} \leq c_{02} \beta t
$$

i.e., $\left|x_{0}(t)\right| \leq c_{02} \beta t$, for all $t>0$. At the same time

$$
\begin{aligned}
\frac{d V_{0}}{d t} & =2 x_{0}\left(d_{0}(t) u_{0}+x_{0} \phi_{0}^{d}\left(t, x_{0}\right)\right) \\
& \geq 2 c_{01} \beta x_{0}-2 x_{0}^{2}\left(1+\frac{c_{02}}{c_{01}}\right) \phi_{0}\left(x_{0}\right) \\
& =2 c_{01} \beta \sqrt{V_{0}}-2\left(1+\frac{c_{02}}{c_{01}}\right) \phi_{0}\left(x_{0}\right) V_{0} .
\end{aligned}
$$

Then, using the variable coefficient method, we have

$$
\begin{aligned}
V_{0}(t) & \geq\left(c_{01} \beta\right)^{2} e^{-2 \mu(t)}\left(\int_{0}^{t} e^{\mu(\tau)} d \tau\right)^{2} \\
& \geq\left(c_{01} \beta\right)^{2} e^{-2 \mu(t)} t^{2}
\end{aligned}
$$

where $\mu(t)=\left(1+\left(c_{02} / c_{01}\right)\right) \int_{0}^{t} \phi_{0}\left(x_{0}(s)\right) d s \geq 0$ for all $t$. Then, $V_{0}(t)>0$ for all $t>0$.

Proof of Theorem 3: If $\left(x_{0}(0), x(0)\right)=(0,0)$ and $\left(x_{0}(0), x(0)\right) \notin \Gamma \cup\{(0,0)\}$, Theorem 3 is a direct consequence of Theorem 1 . So we only need to consider the case when $\left(x_{0}(0), x(0)\right) \in \Gamma$.

First, the following inequality is satisfied:

$$
u_{0}(t) \geq \frac{\beta}{2}, \quad t<t_{s}^{1}(|x(0)|)
$$

where $t_{s}^{1}(|x(0)|)=\min \left\{\left(c_{01} /\left(2 c_{02} T_{0}\left(c_{02} \beta|x(0)|\right)\right)\right),|x(0)|\right\}$. Indeed, from Lemma 3 , it follows that $\left|x_{0}(t)\right| \leq c_{02} \beta t_{s}^{1}(|x(0)|)$ when $t<t_{s}^{1}(|x(0)|)$. Then

$$
\begin{aligned}
u_{0}(t) & =\beta-x_{0}(t) \frac{\phi_{0}\left(x_{0}(t)\right)}{c_{01}} \\
& \geq \beta-c_{02} \beta t_{s}^{1}(|x(0)|) \frac{T_{0}\left(c_{02} \beta|x(0)|\right)}{c_{01}} \\
& \geq \frac{\beta}{2} \quad \forall t<t_{s}^{1}(|x(0)|) .
\end{aligned}
$$

Second, from Lemma 3 we know that $x_{0}\left(t_{s}\right) \neq 0$. So we can switch from $u_{0}(t)=\beta-\left(\phi_{0} / c_{01}\right) x_{0}$ when $t<t_{s}(|x(0)|)$ to $u_{0}(t)=-\left(\lambda_{0}+\right.$ $\left.\left(\phi_{0} / c_{01}\right)\right) x_{0}$ when $t \geq t_{s}(|x(0)|)$.

Third, in order to employ the controller $u$ obtained in Theorem 1, we should verify that there exists $\varphi_{0}\left(x_{0}\right)$ such that

$$
\left|\frac{\dot{u}_{0}}{u_{0}}\right| \leq \varphi_{0}\left(x_{0}\right) \quad \text { for } t<t_{s}(|x(0)|)
$$

It is easy to know that

$$
\dot{u}_{0}=-\left(\frac{1}{c_{01}} \phi_{0}\left(x_{0}\right)+\frac{1}{c_{01}} x_{0} \phi_{0}^{\prime}\left(x_{0}\right)\right)\left(d_{0} u_{0}+x_{0} \phi_{0}^{d}\right) .
$$


So

$$
\begin{aligned}
\left|\frac{\dot{u}_{0}}{u_{0}}\right| \leq\left(c_{02}+\frac{1}{\beta}\left(x_{0}^{2}\right.\right. & \left.\left.+\phi_{0}^{2}\left(x_{0}\right)\right)\right) \\
& \times\left(\frac{1}{c_{01}} \phi_{0}\left(x_{0}\right)+\frac{1}{2 c_{01}}\left(x_{0}^{2}+\left(\phi_{0}^{\prime}\left(x_{0}\right)\right)^{2}\right)\right) .
\end{aligned}
$$

In view of the facts that

$$
\left|x_{0}(t)\right| \leq \begin{cases}c_{02} \beta|x(0)|, & \text { if } t<t_{s}(|x(0)|) \\ c_{02} \beta|x(0)| e^{-\lambda_{0}\left(t-t_{s}\right)}, & \text { if } t \geq t_{s}(|x(0)|)\end{cases}
$$

and

$$
V\left(x_{0}(t), x(t), u_{0}(t)\right) \leq V\left(x_{0}(0), x(0), u_{0}(0)\right) e^{-2 \lambda t}
$$

the closed-loop system is globally $\mathcal{K}$-exponentially stable at the origin.

Remark 1: It should be emphasized that a feedback controller may become excessively large even for small states. In particular, this may happen for initial conditions close to a singular manifold as in [6] and [10]. The key feature of our proposed feedback laws is that the controller $\beta-\left(\phi_{0} / c_{01}\right) x_{0}(t)$ is applied only as $x_{0}(0)$ is zero and $x(0) \neq 0$ in order to retrieve "some sufficient" controllability on the state $x$. Note that along the trajectories of the closed-loop system, when $u_{0}$ tends to zero, the state does the same.

Remark 2: In the absence of input and state disturbances, (1) becomes the standard chained system (i.e., $d_{i}=1, \phi_{i}^{d}=0,0 \leq i \leq$ $n-1)$. An exponentially stabilizing controller was recently obtained in [15], based on a Riccati equation. However, it is difficult, if not impossible, to extend the algorithm of [15] to the case when a chained system is subject to disturbances as in (1).

\section{EXAMPLE}

A tricycle-type mobile robot with nonholonomic constraints on the linear velocity has often been used as a benchmark example in the recent literature on nonholonomic control systems design [8], [10]. In [8], Morse et al. addressed the parking problem for the mobile robot of unicycle type in the presence of parametric uncertainties

$$
\left\{\begin{array}{l}
\dot{x}=p_{1}^{*} v \cos \psi \\
\dot{y}=p_{1}^{*} v \sin \psi \\
\dot{\psi}=p_{2}^{*} \omega
\end{array}\right.
$$

where $(x, y)$ denotes the position of the center of mass of the robot, $\psi$ is the heading angle of the robot, $v$ is the forward velocity, $\omega$ is the angular velocity of the robot, and $p_{1}^{*}$ and $p_{2}^{*}$ are (unknown) positive parameters determined by the radius of the rear wheels and the distance between them.

The problem addressed in [8] and [10] was to steer the robot to the origin by a state-feedback control law, regardless of the value of the unknown constant parameters $p_{1}^{*}$ and $p_{2}^{*}$. In [8], a supervisory control scheme was presented to solve the problem without a priori knowledge of the parameters $p_{1}^{*}$ and $p_{2}^{*}$. However, the convergence rate is not exponential but asymptotic. In [10], using the following change of coordinates and feedback:

$$
\begin{aligned}
x_{0} & =\psi \\
x_{1} & =x \sin \psi-y \cos \psi \\
x_{2} & =x \cos \psi+y \sin \psi \\
u_{0} & =\omega \\
u & =v
\end{aligned}
$$

(21) was transformed into the following form:

$$
\left\{\begin{array}{l}
\dot{x}_{0}=p_{2}^{*} u_{0} \\
\dot{x}_{1}=p_{2}^{*} x_{2} u_{0} \\
\dot{x}_{2}=p_{1}^{*} u-p_{2}^{*} x_{1} u_{0} .
\end{array}\right.
$$

Then, a switching control scheme was presented to solve the problem without a priori knowledge of the parameters $p_{1}^{*}$ and $p_{2}^{*}$ in [10]. The closed-loop system is not Lyapunov stable although the convergence rate is exponential.

We will design a robust state-feedback controller to drive the states of (21) to the origin with exponential convergence and Lyapunov stability. Introducing the variables

$$
\xi_{1}=\frac{x_{1}}{u_{0}} \quad \xi_{2}=x_{2}
$$

then

$$
\left\{\begin{array}{l}
\dot{x}_{0}=p_{2}^{*} u_{0} \\
\dot{\xi}_{1}=p_{2}^{*} \xi_{2}-\xi_{1} \frac{\dot{u}_{0}}{u_{0}} \\
\dot{\xi}_{2}=p_{1}^{*} u-p_{2}^{*} \xi_{1} u_{0}^{2}
\end{array}\right.
$$

So, the following controller can be obtained.

- $x_{0}(0)=0, x(0)=\left(x_{1}(0), x_{2}(0)\right) \neq(0,0)$, see the equation shown at the bottom of the page, where $t_{s}(|x(0)|)=\min \left\{\delta,\left(p_{2 \max } /\left(2 \epsilon p_{1 \min }\right)\right),|x(0)|\right\}, \epsilon>0$, $\lambda_{0}>0, \beta>0, \lambda_{1}>\left(1 / p_{1 \min }\right), \lambda_{2}>0, \lambda_{3}>\lambda_{0}+\left(1 / p_{2 \min }\right)$, and $\lambda_{4}>0$.

- $\left(x_{0}(0), x(0)\right)=(0,0)$,

$$
u_{0}=0 \quad u=0 .
$$

- $x_{0}(0) \neq 0$

$$
\begin{aligned}
u_{0}=- & \lambda_{0} x_{0} \\
u=- & \left(\lambda_{4}+\frac{p_{2 \max }^{2}}{4 p_{1 \min }}\left(1-\lambda_{0}^{2} x_{0}^{2}-\lambda_{3}^{2}+\lambda_{3} \lambda_{0}\right)^{2}\right. \\
& \left.\quad+\lambda_{3} \frac{p_{2 \max }}{p_{1 \min }}\right)\left(\xi_{2}+\lambda_{3} \xi_{1}\right)
\end{aligned}
$$

where $\lambda_{0}>0, \lambda_{3}>\lambda_{0}+\left(1 / p_{2 \min }\right), \lambda_{4}>0$.

$$
\begin{aligned}
& u_{0}(t)= \begin{cases}\beta, & \text { if } t<t_{s}(|x(0)|) \\
-\lambda_{0} x_{0}, & \text { if } t \geq t_{s}(|x(0)|)\end{cases} \\
& u(t)= \begin{cases}-\left(\lambda_{2}+\frac{p_{2 \max }^{2}}{4 p_{1 \min }}\left(1+\beta^{2}+\lambda_{1}^{2}\right)^{2}+\lambda_{1} \frac{p_{2 \max }}{p_{1 \min }}\right)\left(\xi_{2}+\lambda_{1} \xi_{1}\right), & \text { if } t<t_{s}(|x(0)|) \\
-\left(\lambda_{4}+\frac{p_{2 \max }^{2}}{4 p_{1 \min }}\left(1-\lambda_{0}^{2} x_{0}^{2}-\lambda_{3}^{2}+\lambda_{3} \lambda_{0}\right)^{2}+\lambda_{3} \frac{p_{2 \max }}{p_{1 \min }}\right)\left(\xi_{2}+\lambda_{3} \xi_{1}\right), & \text { if } t \geq t_{s}(|x(0)|)\end{cases}
\end{aligned}
$$



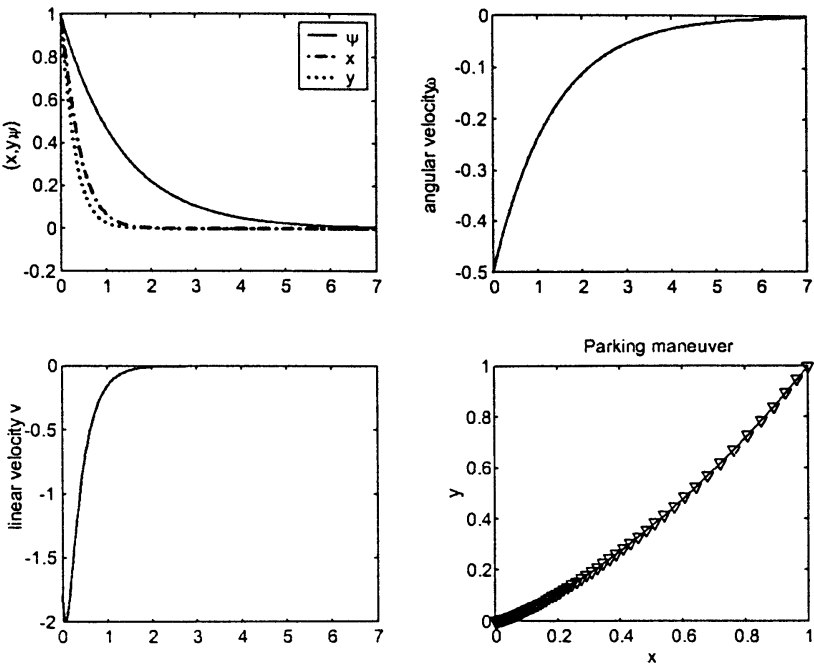

Fig. 1. Closed-loop signals with initial condition $(\boldsymbol{x}(0), \boldsymbol{y}(0), \boldsymbol{\psi}(0))=$ $(1,1,1)$.
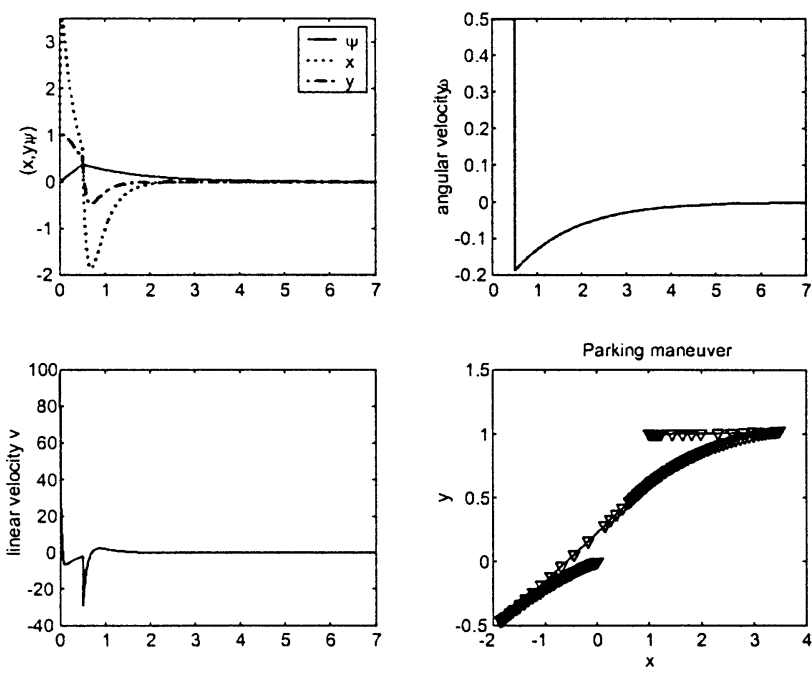

Fig. 2. Closed-loop signals with initial condition $(x(0), y(0), \psi(0))=$ $(0,1,1)$.

Our simulations as shown in Figs. 1 and 2 are based on the following choice of design and system parameters [10]:

$$
\begin{aligned}
p_{1 \min } & =p_{2 \min }=1 \quad p_{1 \max }=p_{2 \max }=2 \\
p_{1}^{*} & =p_{2}^{*}=1.5 \quad \lambda_{0}=0.5 \quad \lambda_{1}=2 \quad \lambda_{2}=1 \\
\lambda_{3} & =2 \quad \lambda_{4}=1 \quad \epsilon=1 \quad \beta=0.5 \quad \delta=1 .
\end{aligned}
$$

It can be clearly seen from the simulation results that exponential convergence is achieved.

\section{CONCLUSION}

In this note, the problem of global $\mathcal{K}$-exponential stabilization is considered for a class of nonholonomic chained systems with strongly nonlinear input/state driven disturbances and drifts. Using input-state scaling and backstepping techniques, a globally exponentially convergent state-feedback control law is designed. Using a switching scheme dependent on the initial condition, Lyapunov stability and exponential convergence are guaranteed for the closed-loop system. The simulations results in a wheeled mobile robot have demonstrated the effectiveness of the proposed control design approach.

\section{ACKNOWLEDGMENT}

The authors would like to thank the Associate Editor and the Reviewers for a number of constructive comments based on which this has been greatly improved.

\section{REFERENCES}

[1] A. Astolfi, "Discontinuous control of nonholonomic systems," Syst. Control Lett., vol. 27, pp. 37-45, 1996.

[2] A. Astolfi and W. Schaufelberger, "State and output feedback stabilization of multiple chained systems with discontinuous control," in Proc. 35th IEEE Conf.Decision and Control, Kobe, Japan, 1996, pp. 1443-1447.

[3] A. Bloch, M. Reyhanoglu, and N. H. McClamroch, "Control and stabilization of nonholonomic dynamic systems," IEEE Trans. Automat. Contr., vol. 37, pp. 1746-1757, Nov. 1992.

[4] R. W. Brockett, "Asymptotic stability and feedback stabilization," in Differential Geometric Control Theory, R. W. Brockett, R. S. Millman, and H. J. Sussman, Eds., 1983, pp. 181-191.

[5] C. Canudas de Wit, B. Siciliano, and G. Bastin, Theory of Robot Control, 3rd ed. London, U.K.: Springer-Verlag, 1996.

[6] K. D. Do and J. Pan, "Adaptive global stabilization of nonholonomic systems with strong nonlinear drifts," Syst. Control Lett., vol. 46, pp. 195-205, 2002.

[7] M. Fliess, J. Levine, P. Martin, and P. Rouchon, "Flatness and defect of nonlinear systems: Introductory theory and examples," Int. J. Control, vol. 61, pp. 1327-1361, 1995.

[8] J. P. Hespanha, S. Liberzon, and A. S. Morse, "Logic-based switching control of a nonholonomic system with parametric modeling uncertainty," Syst. Control Lett., vol. 38, no. 3, pp. 167-177, 1999.

[9] Z. P. Jiang, "Iterative design of time-varying stabilizers for multi-input systems in chained form," Syst. Control Lett., vol. 28, pp. 255-262, 1996.

[10] — "Robust exponential regulation of nonholonomic systems with uncertainties," Automatica, vol. 36, pp. 189-209, 2000.

[11] I. Kolmanovsky and N. H. McClamroch, "Developments in nonholonomic control problems," IEEE Control Syst. Mag., vol. 15, pp. 20-36, June 1995.

[12] M. Krstić, I. Kanellakopoulos, and P. V. Kokotović, Nonlinear and Adaptive Control Design. New York: Wiley, 1995.

[13] W. Lin, R. Pongvuthithum, and C. Qian, "Control of higher-order noholonomic systems in power chained form by discontinuous feedback," IEEE Trans. Automat. Contr., vol. 47, pp. 108-115, Jan. 2002.

[14] J. Luo and P. Tsiotras, "Exponentially convergent control laws for nonholonomic systems in power form," Syst. Control Lett., vol. 35, pp. 87-95, 1998.

[15] N. Marchand and M. Alamir, "Discontinuous exponential stabilization of chained form systems," Automatica, vol. 39, pp. 343-348, 2003.

[16] R. M'Closkey and R. Murray, "Exponential stabilization of driftless nonlinear control systems using homogeneous feedback," IEEE Trans. Automat. Contr., vol. 42, pp. 614-628, May 1997.

[17] P. Morin, J. B. Pomet, and C. Samson, "Developments in time-varying feedback stabilization of nonlinear systems," in Proc. Nonlinear Control Systems Design Symp. (NOLCOS'98), vol. 1/2, 1998, pp. 587-594.

[18] R. M. Murray and S. Sastry, "Nonholonomic motion planning: Steering using sinusoids," IEEE Trans. Automat. Contr., vol. 38, pp. 700-716, May 1993.

[19] J. B. Pomet, "Explicit design of time-varying stabilizing control laws for a class of controllable systems without drift," Syst. Control Lett., vol. 18, pp. $147-158,1992$.

[20] O. J. Sordalen and O. Egeland, "Exponential stabilization of nonholonomic chained systems," IEEE Trans. Automat. Contr., vol. 40, pp. 35-49, Jan. 1995. 